\title{
Planen für Praktiken. Alltagsbezüge in planerischen Abwägungen am Beispiel von urbanem Grün
}

\section{Planning for practices. Everyday issues in planning considerations using urban green as an example}

https://doi.org/10.2478/rara-2019-0017

Eingegangen: 15. April 2018; Angenommen: 6. Februar 2019

Zusammenfassung: In planungswissenschaftlichen wie auch in gesellschaftlichen Diskursen wird die Rolle der Planung als Entscheidungsträgerin über die Stadtentwicklung zunehmend infrage gestellt. Proteste von Bürgern, seien sie medial kommuniziert oder im öffentlichen Raum ausgetragen, verdeutlichen, dass ihre Belange zu oft vernachlässigt werden. Es zeichnet sich ab, dass ein Umdenken erforderlich ist hin zu einer Stadtentwicklung, die sich stärker nach den Belangen der Bürgerschaft richtet. Hierzu braucht es eine veränderte Planungspraxis, die die Belange der Bürgerschaft in den Mittelpunkt stellt. Gleichzeitig sind Ansätze und Methoden gesucht, mit denen ihre vielfältigen Belange in den Planungsprozess eingebracht werden können. Hierzu greift der Beitrag auf praxistheoretische Annahmen zurück und betrachtet Praktiken der Raumproduktionen als Zugang zu alltagsweltlichen Belangen der Bürgerschaft, die es planerisch zu bedienen gilt. Am Beispiel von städtischem Grün wird aufgezeigt, wie die Analyse sozialer Praktiken ein differenziertes Bild alltagsweltlicher Relevanzsetzungen aufzeigen kann, das planerischen Abwägungen Orientierungen bietet.

Schlüsselwörter: Praktiken, Raumproduktion, Partizipation, Walking interview, Stadtgrün, Bürgerbelange

Abstract: Discourses in planning theory and in society question the role of planning as decision makers for urban development. Protests of citizens show that their concerns often find too little attention in questions of developing cities. In consequence, a need for rethinking the practice of urban planning becomes apparent. Therefore, it is required to implement an understanding of planning as an integrative process, which is informed by the needs of the people living in the city. At the same time, methods and concepts are needed to identify the heterogeneous concerns of the citizens and to bring them into the planning process. In the first part, the paper presents an idea of citizen-oriented planning practice. In the second part, a methodological framework shows how the differentiated needs of people can be identified and abstracted as a framework which serves as an orientation for decision-making in planning processes. Therefore, the paper refers to approaches of practice theory and focusses on everyday productions of space as a heuristic tool for identifying the needs of people practicing their everyday life. For making it more concrete, the concept is adapted to questions of planning the urban green. Empirical findings show how

${ }^{*}$ Corresponding author: Dr. Jana Kühl, Christian-Albrechts-Universität zu Kiel, Geographisches Institut, Ludewig-Meyn-Straße 14, 24098 Kiel, Deutschland, E-mail: kuehl@geographie.uni-kiel.de 
urban green typically gets relevant in people's practices of everyday life and how planning practice can refer to these findings seeking for a citizen driven urban development.

Keywords: Practices, Production of space, Participation, Walking interview, Urban green, Civic involvement

\section{Einleitung}

Ob Großprojekte, die in Verruf geraten, oder Bauvorhaben im Kleinen, die in der Nachbarschaft Unmut erzeugen - immer wieder werden planerische Vorhaben von Protesten begleitet, in denen Betroffene eines Planungsvorhabens ein Vetorecht einfordern und über Stadtentwicklungsfragen mitentscheiden wollen (Menzl 2014; Gribat/Kadi/Lange et al. 2017). Derartige Proteste zeigen, dass sich Bürger in ihren Anliegen gegenüber der Planung vernachlässigt oder übergangen sehen. In der Folge regt sich Unverständnis und Unzufriedenheit über planerische Vorhaben, bis hin zu einer wachsenden Frustration und einem Gefühl der Entfremdung des eigenen Lebensumfeldes (Diebäcker 2008: 274). Dabei scheinen die Proteste zugleich eine neue Dimension anzunehmen: Dank der Informationsangebote neuer Medien ist die Bevölkerung öfter gut über planerische Vorhaben informiert. Auch bilden sich mittels digitaler Kommunikationsmedien Interessengruppen, die sich medial vernetzen und sich für ihre Anliegen einsetzen (Wiechmann/Terfrüchte 2013: 27 f.). So gerät die öffentliche Hand, nicht zuletzt auch durch die gesellschaftliche Wirkmacht der Medien und sozialer Netzwerke, unter Rechtfertigungsdruck ihrer planerischen Entscheidungen. Mit der Formierung derartiger Konflikte drängt sich der Eindruck auf, dass die angewandten Planungspraktiken den Anliegen der Bürgerschaft nicht ausreichend gerecht werden.

Die Gründe für die Divergenz zwischen planerischen Entscheidungen und den Anliegen der Bürgerschaft können vielfältig sein. Sei es, dass die Belange der Bürgerschaft in den vollzogenen Planungspraktiken nicht ausreichend ermittelt wurden, oder aber, dass andere Belange, wie etwa Wirtschaftsinteressen und politische Strategien, schwerer wogen. Ebenso lässt sich fragen, ob überhaupt ausreichend Kenntnis über die Belange der Bürgerschaft erworben werden konnten, um diese in planerischen Abwägungen einzubringen. In gängigen formalen wie informellen Partizipationsverfahren beteiligt sich in der Regel nur ein kleiner, nicht repräsentativer Ausschnitt der Bevölkerung. Oft dominieren artikulationsstarke Personen den Prozess, während der Austausch zwischen Planenden und Bürgerschaft auf die Abhandlung von Einzelinteressen beschränkt bleibt.
Ebenso verfügen nicht alle Bürger gleichermaßen über die Fähigkeiten und die Möglichkeiten, ihre Belange in Planungsprozessen zu verhandeln, wodurch eine Marginalisierung von Belangen weniger präsenter sozialer Gruppen durch die sozial selektive Wirkung von Partizipationsverfahren noch verstärkt wird (vgl. Lepofsky/ Fraser 2003). Gleichzeitig sind in der räumlichen Planung Zielgruppendefinitionen etabliert, die Menschen anhand ihrer soziodemographischen Merkmale Bedarfe zuschreiben (z. B. Migranten, Frauen). Durch diese Fremdzuschreibung werden Stereotype von Bedarfsmustern hervorgebracht und reproduziert, die dem tatsächlichen Spektrum an Bedarfen der betroffenen Menschen meist nicht gerecht werden (Huning 2014: 34).

Der Beitrag greift die Divergenz zwischen Belangen der Bürgerschaft und planerischen Entscheidungen auf. Er stellt einen theoretischen wie auch einen empirischen Zugang zur Diskussion, mit dem Belange der Bürgerschaft umfassend erschlossen werden können, um sie für planerische Abwägungen zugunsten einer stärkeren Integration von Belangen der Bürgerschaft in der Planung zugänglich zu machen. Möchte man der Bestrebung folgen, einen Einklang zwischen Erfordernissen der Stadtentwicklung und den Belangen der Bürgerschaft herzustellen, braucht es ein verändertes Selbstverständnis der Planungspraxis, wie planerische Lösungen zustande kommen.

Hierzu verweist der vorliegende Beitrag in Kapitel 2 auf planungstheoretische Konzeptionen einer Planungspraxis, die sich den Belangen der Bürgerschaft verpflichtet. In Kapitel 3 wird dargelegt, wie sich diese Belange umfassend ermitteln lassen. Dabei richtet sich der Fokus auf einen städtischen Kontext, da sich gerade hier Dynamiken und Konflikte in der planerischen Entwicklung des begrenzten Raums entfesseln. Nach einer kurzen Ausführung praxistheoretischer Grundannahmen (Kapitel 3.1 und 3.2) folgt die Darstellung eines Ansatzes, mit dem die Perspektiven der Bürgerschaft auf einen Planungsraum anhand konkret vollzogener Praktiken erschlossen werden können (Kapitel 3.3). Um dabei Belange der Bürgerschaft in ihrer Raumbezogenheit herauszustellen, werden diese als Resultat von Praktiken der Raumproduktion konzeptionell konkretisiert. Hierauf aufbauend wird ein Erhebungskonzept vorgeschlagen, mit dem sich herausarbeiten lässt, wie sich Menschen 
einen Planungsraum praktisch aneignen und was innen dabei wichtig ist. Damit wird zugleich die Idee einer Planung aufgeworfen, die für die alltäglichen Praktiken der Bevölkerung und den hierbei verfolgten Relevanzsetzungen einen geeigneten Rahmen schafft (Kapitel 3.4). Zur Verdeutlichung dieses Zugangs schließt der Beitrag in Kapitel 4 mit dem Beispiel einer empirischen Studie zur Relevanz städtischen Grüns, in der die Ermittlung alltagsweltlicher Relevanzsetzungen für planerische Schlüsse zur Anwendung kommt. Welches komplexe Forschungsfeld der schwierige Prozess, die oft widersprüchlichen Belange abzuwägen, eröffnet, wird im Fazit (Kapitel 5) umrissen.

\section{Planungsverständnisse im Interesse der Bürgerschaft}

Aus der Sicht der Stadtplanung sind Städte primär Funktionsräume, die es auszuformen gilt. Doch werden diese Funktionsräume erst zu Städten im gesellschaftlichen Sinne, wenn sich Menschen den Stadtraum zu eigen machen, sich dort aufhalten, wohnen, arbeiten und konsumieren und die Funktionsräume so auf eine eigene, oft ungeplante Weise ausfüllen. So verleihen erst die Menschen und ihre Aktivität dem Stadtraum ihre gesellschaftliche Funktion wie auch ihre Wirtschaftskraft (vgl. Gehl 2010; Brenner/Marcuse/Mayer 2012). Folgt man dieser Betrachtung von Städten als Lebensräume, erscheint es plausibel, die Planung von Städten an den Bedürfnissen jener Menschen auszurichten, die in Städten leben und sie beleben. Im Wettbewerb um Einwohner und Unternehmensansiedlungen besteht spätestens seit Florida (2004) ein Bewusstsein dafür, Städte zu attraktiven Lebensräumen zu entwickeln. Doch werden in der Praxis nicht selten die Bedürfnisse der Bewohner sowie der Besucher verfehlt. Durch Prozesse der Neoliberalisierung wird die Stadtentwicklung zunehmend von einer marktwirtschaftlichen Logik dominiert, während die öffentliche Hand infolge kommunaler Haushaltsdefizite geneigt ist, sich Investorenbelangen anzupassen (Deutscher Städtetag 2013: 7). Diese Tendenzen werfen die Frage auf, wie Planende in Aushandlung mit Belangen der Bürgerschaft treten. Für wen plant die Planung und welche Interessen finden Berücksichtigung?

In der Planungstheorie werden derartige Aushandlungen als Gegenstand von „Recht-auf-Stadt"-Bewegungen betrachtet, die auf einer neomarxistischen Haltung gründen, wie sie vor allem durch Lefèbvre (1996) und Harvey (2008) geprägt wurde. Hiermit einher gehen For- derungen nach einer partizipativen und sozial gerechten Ausgestaltung planerischer Entscheidungen. Friedmann (2010: 159) formuliert mit Bezugnahme auf Lefèbvre (1996) eine moralische Verantwortung der Planung, der Bürgerschaft ein Gestaltungsrecht für ihre Nachbarschaft zu gewährleisten: „Contrary to command planning, which globally speaking is still the dominant form, I would argue that planners need directly to engage those who reside in neighborhoods, and that this engagement means to establish a moral relation that from the start acknowledges people's ,right to the city' which is to say their right to local citizenship [...]." In eine ähnliche Richtung verweisen die Bestrebungen zur Verwirklichung einer integrativen, sozial gerechten Planung, wie sie unter dem Schlagwort der Just City gefordert wird (Harvey 2009; Fainstein 2010; Brenner/Marcuse/ Mayer 2012). Diesem normativen Verständnis folgend ist Planung eine politische Aufgabe, die sich gesellschaftlicher Prozesse annehmen und dabei nach einem Ausgleich und nach sozialer Gerechtigkeit streben sollte (Gribat/Kadi/Lange et al. 2017: 11 f.).

Die Forderung nach einer Planung, die sich näher an den Belangen der Bürgerschaft vollzieht, ist keineswegs neu. Bereits in den 1970er-Jahre kritisierte etwa Burckhardt eine Planungspraxis, die an den Bedarfen der Bevölkerung vorbeiplante und stattdessen unwirtliche Stadträume entstehen ließ (vgl. Burckhardt 2004). Ebenso wurde eine Planungspraxis kritisiert, die die Sichtweisen der Bürgerschaft als Laienperspektive ohne Erkenntniswert diskreditiert und in der planerische Entscheidungen über die Expertise der Planenden legitimiert werden (Selle 2007; Heinemann/Selle/ Sutter-Schurr 2008; Tessin 2008). Im Hinblick auf immer wieder auftretende Konflikte, in denen sich Bürger übergangen sehen, hat diese Kritik an gängigen Planungspraktiken auch heute Bestand. So verweisen die kritischen Stimmen aus der Gesellschaft wie auch aus den Planungswissenschaften auf die Notwendigkeit, etablierte Planungspraktiken zu überdenken (vgl. Bock/ Reimann 2012). Dieser Herausforderung folgend formulieren planungswissenschaftliche Ansätze der kommunikativen Planung die Vorstellung, dass Planer stärker in den Dialog treten (sollen) und Planung in Kollaboration mit verschiedenen Interessengruppen gestalten (z. B. Healey 1992; Fischer/Gottweis 2012). Darüber hinaus fordert Healey (2010) mit inrem Konzept der kollaborativen Planung die Akteure der räumlichen Planung auf, Städte lebenswerter zu gestalten, indem sie die vielfältigen Belange sozialer Akteure zusammentragen und hieraus einen Konsens einer angemessenen Stadtentwicklung ableiten. Dabei gewinnen Vorstellungen einer 
deliberativen Planung an Bedeutung, in der ein Austausch mit der Bürgerschaft gesucht wird (Healey 1997). Einen Schritt weiter gehen Ansätze der partizipativen Forschung, in der zivile Akteure selbst planerische Problemstellungen identifizieren und gemeinsam mit Akteuren der Planung Lösungen erarbeiten (Bergold/Thomas 2010).

Ein auf Dialog und Kollaboration gerichteter Stadtentwicklungsprozess erfordert ein planerisches Selbstverständnis, in dem die Bürgerschaft als Erkenntnisquelle für planerische Fragen konsultiert wird. Wesentliche Belange der Stadtentwicklung zeigen sich erst aus einer Perspektive derjenigen, die im Stadtraum ihren Alltag gestalten. Die Menschen vor Ort sind vertraut mit Störfaktoren und Unstimmigkeiten im Stadtraum, ebenso mit lokalspezifischen Besonderheiten, die Stadträume als lebenswert konstituieren. Hierbei ist nicht nur die physische Ausstattung relevant, sondern vor allem auch emotionale und affektive Verbindungen zu dem vertrauten Umfeld, die sich auch als Sense of Place beschreiben lassen (vgl. Kovács/Musterd 2013: 98 ff.). Das Präferieren wie auch die Ablehnung bestimmter Orte haben Einfluss darauf, wo sich Menschen wie bewegen, welchen Räumen sie sich verbunden fühlen und ob Menschen für die Gemeinschaft aktiv werden. Auch können raumbezogene Zuschreibungen planungsrelevante Auswirkungen haben, wie etwa die Bevorzugung oder Ablehnung bestimmter Wohnlagen. Um diese Kenntnisse für die planerische Entwicklung lebenswerter Stadträume nutzen zu können, ist demnach in der Planungspraxis stärker darauf zu achten, wie Menschen ihre Umwelt erleben, sie empfinden und sie sich schließlich individuell zu eigen machen (Healey 2010: 8). Damit eröffnet sich sogleich ein Weg, den Bedarfen der Bürgerschaft als Betroffene planerischer Maßnahmen nachzugehen und gerecht zu werden (Gerlich/Stoik 2008: 253).

Jedoch - ebenso differenziert, wie sich die Gegenwartsgesellschaft zeigt, sind auch die Ansprüche und Vorstellungen von einer lebenswerten Stadt, die es zu berücksichtigen gilt (Healey 2010: 31). So sind Mittel und Wege gesucht, um die Diversität der Belange in strukturierter Weise zu erfassen und zur Abwägung zu bringen. Offen bleibt, wie die Ermittlung der Belange der Bevölkerung gelingt. Hier bieten praxistheoretische Ansätze einen Zugang, um Belange zu ermitteln, wie sie sich in unterschiedlichster Form aus der praktischen Alltagsgestaltung ergeben.

\section{Praktiken als Orientierung pla- nerischer Praxis}

In der sozial- und kulturwissenschaftlichen Forschung haben praxistheoretische Ansätze einen Theorieansatz eingebracht, mit dem sich der Fokus auf praktische Tätigkeiten sozialer Akteure richtet. Dieser Fokus wird zunehmend auch in den Planungswissenschaften als erkenntnisträchtig aufgegriffen. Da sich die Planungswissenschaften gerade der Tätigkeit des Planens widmen, wundert es nicht, dass die Begriffe „Praxis“ und „Praktiken“ hier gängige Konzepte bilden. Mehr noch scheinen die Begriffe so etabliert, dass sie selten eine konzeptionelle Aufarbeitung erfahren. Dabei bezieht sich der Begriff der Praxis in den Planungswissenschaften im Allgemeinen auf die Planungspraxis und meint damit planerische Tätigkeiten, wie sie für Gebietskörperschaften vollzogen werden. Die Thematisierung der Praxis dient zum einen als Kontrastfolie gegenüber den theoretischen Ausarbeitungen der Planungswissenschaften (z. B. Diller/Thaler 2017; Zibell 2017): Mit der Gegenüberstellung von Wissenschaft und Praxis werden Fragen der Anschlussfähigkeit planungswissenschaftlicher Erkenntnisse für den Alltag der räumlichen Planung thematisiert sowie Forschungsbedarfe aus dem Planungsalltag heraus identifiziert. Zum anderen wird die Praxis selbst als Gegenstand der Planungswissenschaften herangezogen (vgl. z. B. Lange/Müller 2016). Hierunter wird thematisiert, wie Planung optimalerweise prozessual oder nach normativen Kriterien auszugestalten ist. Auch wird der gegenstandsbezogene Umgang mit Technologien, Themen und Metadiskursen der Planungspraxis behandelt, wie z. B. im Handlungsfeld der Windenergie. Nicht zuletzt wird hinsichtlich der Planungspraxis das (Selbst-) Verständnis von Planung um die Rolle der Planenden reflektiert (Levin-Keitel/Othengrafen 2016). Hierunter ermöglicht die Reflexion gängiger Planungspraktiken, den Umgang mit Belangen der Bürgerschaft zu optimieren.

Alle benannten Zugänge streben danach, Praktiken des Planens zu identifizieren und zu beschreiben. Teils bewerten sie auch die identifizierten Praktiken und zeigen Bedarfe und Strategien auf, Planungspraktiken zu verändern. Demgegenüber wurden Praktiken als Gegenstand von Planung bislang nicht explizit thematisiert. Gemeint sind jene Praktiken, mit denen Menschen ihren Altag gestalten. Hier eröffnet der Blick auf alltägliche Praktiken Erkenntnisse über Bedarfe, wie sie sich für Menschen innerhalb eines Planungsraumes in der praktischen Alltagsgestaltung ergeben. Für die Menschen einer Stadt ist 
vordergründig von Interesse, dass die planerischen Lösungen, so wie sie sie im Stadtraum vorfinden, ihren Bedarfen und Vorstellungen gerecht werden. Ist bekannt, wie Menschen mit dem im Stadtraum Vorgefundenen umgehen und wie sie ihren Alltag praktisch ausgestalten (möchten), so lassen sich hieraus Lösungen für die räumliche Planung ableiten. Damit gelingt eine Perspektivenschärfung auf alltagsweltliche Belange mithilfe von praxistheoretischen Ansätzen.

\subsection{Grundlagen eines praxistheoreti- schen Zugangs}

Die Bezeichnung Praxistheorie (auch Praxeologie oder Praktikentheorie) dient als Oberbegriff, unter dem sich poststrukturalistische und posthumanistische Theorieansätze sowie Ansätze der Ethnomethodologie und Cultural Studies finden lassen (Reckwitz 2003: 283). Als prägend sind hierbei die Arbeiten von Bourdieu (1976), Schatzki (1996) und Reckwitz (2003) zu nennen. Aus einer praxistheoretischen Perspektive werden Praktiken als kleinste Einheit zur Analyse gesellschaftlicher Zusammenhänge verstanden (Reckwitz 2003: 290). Dabei meint Praktiken das konkrete Tun im Umgang mit Entitäten und sozialen Situationen. Praktiken werden als spezifische Handlungsweisen körperlich vollzogen, wobei das Sprechen über etwas oder mit jemandem gleichermaßen eine Praktik ist, wie jedes andere körperlich vollzogene Tun (Schatzki 1996: 89). Menschen setzen sich im Tun mit ihrer sozialen und dinglichen Umwelt auseinander und lernen, mit ihr auf eine sinnhafte Weise umzugehen, wie etwa mit dem Bedienen eines Smartphones oder auch in der Organisation des Alltagslebens. Durch den Umgang mit Entitäten und sozialen Situationen werden Sinnwelten als eine Art persönliche Erfahrungswirklichkeit geformt, in denen der Handelnde seine Umwelt gemäß seinen Erfahrungen auf spezifische Weise deutet und versteht.

Aus dieser konstruktivistischen Perspektive konstituieren sich Stadträume erst in Auseinandersetzung mit Entitäten wie auch in sozialen Interaktionen, mit denen sich Menschen Räume als Teil ihrer Erfahrungswirklichkeit aneignen (Simonsen 2007: 169). Dabei erfolgen die Praktiken, in denen sich Menschen Stadträume aneignen, nicht beliebig. Jede Praktik ist sozial erlernt. Praktiken sind soziale Praktiken, wobei sich das Soziale über kollektive Verhaltensweisen als ein praktisches Können konstituiert: "the social is a field of embodied, materially interwoven practices centrally organized around shared practical understandings" (Schatzki 2001: 12).
Der Vollzug von Praktiken folgt einer sozial geteilten Sinnhaftigkeit und wird als angemessene Handlungsweise, Gepflogenheit oder ,Know-how' gewusst und gekonnt, ohne dass sich der Agierende dessen zwangsläufig bewusst ist. „Beim Vollzug einer Praktik kommen implizite soziale Kriterien zum Einsatz, mit denen sich die Akteure in der jeweiligen Praktik eine entsprechende ,Sinnwelt' schaffen, in denen Gegenstände und Personen eine implizit gewusste Bedeutung besitzen, und mit denen sie umgehen, um routinemäßig angemessen zu handeln" (Reckwitz 2003: 292). Praktiken werden immer in einem bestimmten sozialen Kontext als sinnhaft geformt, womit stets sozialspezifische Gemeinsamkeiten der Ausformung von Erfahrungswirklichkeiten bestehen. Für die Planung bedeutet dies, dass sich überindividuelle Muster finden lassen, nach denen sich Menschen ihren Stadtraum als ihre Erfahrungswirklichkeit aneignen und planungsrelevante Belange aufwerfen. Eben diese Muster zeigen sich in einer praxistheoretischen Perspektive auf den städtischen Alltag.

Wenn das Tun einer verinnerlichten Sinnhaftigkeit folgt, so spiegelt sich diese Sinnhaftigkeit im Umkehrschluss auch im konkreten Tun wider. In der Art und Weise, wie Menschen den Stadtraum wahrnehmen und bewerten, worauf sie ihre Aufmerksamkeit richten und welche Bedarfe sie formulieren, wo sie sich aufhalten, wie sie mit ihren Mitmenschen interagieren, zeigen sich charakteristische Relevanzsetzungen, nach denen Stadträume als Teil alltagsweltlicher Erfahrungswirklichkeiten wahrgenommen und angeeignet werden. Relevanzsetzungen leiten demnach soziale Praktiken an und werden zugleich in innen sichtbar. Eine empirische Differenzierung dieser alltagsweltlicheren Relevanzsetzungen ermöglicht Schlüsse über planerisch relevante Belange, wie sie aus einer alltagsweltichen Sicht auf den Planungsraum aufgeworfen werden. Hierzu sind die Art und Weise des Agierens, Sprach- und Kommunikationsweisen, Handlungsroutinen, Gebrauchspraktiken, Performanzen und anderes, körperlich vollzogenes Tun empirisch aufzuarbeiten, in denen sich die spezifischen Relevanzsetzungen widerspiegeln (Schmidt 2012: 12).

\subsection{Alltägliche Raumproduktionen als Gegenstand der Planung}

Die Rekonstruktion verschiedener Formen des Agierens, in denen Stadträume nach spezifischen Relevanzsetzungen angeeignet werden, bildet ein komplexes Analysefeld. Für eine strukturierte Beschreibung dieses Feldes in der Betrachtung explizit raumbezogener Zusammen- 
hänge ist es hilfreich, die Aneignung von Stadträumen als alltägliche Raumproduktionen aufbauend auf Lefèbvre (1991) zu konzeptualisieren. Nach seinem neomarxistisch geprägten Gesellschaftsverständnis wird Raum fortwährend gesellschaftlich produziert. Stadträume sind ein gesellschaftliches Produkt, das in sozialen Praktiken ausgeformt wird (Lefèbvre 1991: 31). Hierzu gehören Praktiken der Planung ebenso wie Praktiken der Bürgerschaft. Um diese Produktion von (Stadt-)Räumen greifbar zu machen, formuliert Lefèbvre drei Dimensionen der Raumproduktion, die er zugleich aus einer phänomenologischen Perspektive und anhand einer sprachtheoretischen Ableitung spezifiziert (Lefèbvre 1991: 33, 38 f.; vgl. auch Elden 2002: 30). Tabelle 1 listet die drei Dimensionen anhand ihrer Produktionsprozesse auf und ordnet innen die Ausprägungen ihrer Produktion zu. Die Theorie Lefèbvres ist ebenso vielschichtig wie vieldeutig, sodass der nachfolgende Rückgriff auf seine Theorie lediglich als eine mögliche Lesart zu verstehen ist. Diese Lesart zieht die Begrifflichkeiten Lefèbvres heran, um die alltagsweltlichen Aneignungen von Stadträumen beschreibbar zu machen und Interdependenzen zwischen alltagsweltlichen Aneignungsprozessen und der räumlichen Planung aufzuzeigen.

Anlehnend an Lefèbvre (1991: 38) werden Stadträume in räumlicher Praxis materiell geformt. Hierzu zählen das allägliche Tun von Stadtbewohnern wie auch die Tätigkeiten der Planenden, der Architekten oder des Städtebaus. Die materiell produzierten Stadträume dienen sogleich als Ausgangspunkt des wahrgenommenen Raumes, wie er über die Sinne vermittelt in räumlicher Praxis erfasst und angeeignet wird. Diese sinnvermittelte Aneignung von Stadträumen, wie sie sich z. B. im Durchschreiten eines Marktplatzes vollzieht, erfolgt wiederum in Abhängigkeit von diskursiven Konzepten (Repräsentationen des Raumes / Konzipierter Raum) wie auch von Symboliken (Räume der Repräsentation / Gelebter Raum). Aus der Wissensproduktion gehen rational-objektivierende Abstraktionen von Raum als Repräsentationen des Raumes hervor (Lefèbvre 1991: 38 f.). Sie produzieren Wahrheiten, auf die sich Menschen in ihrem Tun beziehen, wie etwa Stadtpläne, Ortsbezeichnungen oder die Definition von Flächennutzungen. Sie prägen als konzipierter Raum die Wahrnehmung des Raumes, indem sie dem Wahrgenommenen sozial geteilte Konzepte zuweisen. Wir erkennen einen Raum als Marktplatz und weisen dem Erkannten bestimmte Attribute zu, wie zum Beispiel Einkauf oder Treffpunkt. Doch wird die Wahrnehmung nicht nur von objektivierten Repräsentationen des Raumes gelenkt. Ebenso werden Räumen Bedeutungen zugewiesen, die aus dem räum- lich verorteten Erlebten hervorgehen. Infolge dieses Erlebens werden Räume zu Räumen der Repräsentation, die symbolisch auf das Erlebte verweisen. Es sind gelebte Räume, so wie sie von dem Einzelnen erfahren wurden (Lefèbvre 1991: 39). So kann ein Marktplatz als Repräsentation einer besonderen Begegnung oder auch in Erinnerung an besondere Gerüche und Geräuschkulissen präsent sein, die den physischen Ort wiederum mit bestimmten Emotionen und Assoziationen belegen. Schließlich wird ein Stadtraum immer zugleich wahrgenommen, konzipiert und erlebt. Die drei Dimensionen sind dialektisch miteinander verbunden und gehen auseinander hervor. Gottdiener (2002: 23) fasst zusammen: „Raum ist gleichzeitig eine räumliche Praxis (eine externalisierte, materielle Umwelt), eine Repräsentation des Raumes (ein konzeptuelles Modell, das eingesetzt wird, um Handlungen zu steuern) und ein Raum der Repräsentation (die gelebte soziale Beziehung der Benutzer zu ihrer Umwelt)."

Die Systematik dreier Dimensionen der Raumproduktion bietet eine Heuristik, mit der sich Belange der Bürgerschaft für einen räumlichen Kontext strukturiert erfassen lassen. Nach einem praxeologischen Verständnis werden dabei die Formen der Ausführung sozialer Praktiken der Raumproduktion betrachtet. In der Art und Weise, wie Räume wahrgenommen, konzipiert und gelebt werden, zeigt sich, wie sich Menschen Planungsräume praktisch zu eigen machen und was innen dabei wichtig ist.

\subsection{Erhebung von Bedarfen in Praktiken der Raumproduktion}

Zur Erhebung der Art und Weise des Praxisvollzugs, in dem Räume (re-)produziert werden, richtet sich der Blick gemäß praxistheoretischer Vorstellungen gleichermaßen auf das Gesagte und das Getane (Schatzki 1996: 89). Im Getanen und Gesagten zeigt sich, „how human practices of everyday life, performance and imagination are implicated in the production of both material and sensory realities and a phenomenological ,sense of place"' (Pink 2008: o. S.). Um jene Praktiken in ihrer Raumbezogenheit zu erheben, eignen sich insbesondere "Walking Interviews“ (vgl. Kühl 2016c). Sie sind eine hybride Form aus teilnehmender Beobachtung und qualitativem Interview und richten einen ethnographischen Blick auf alltägliche Lebenswelten (Evans/Jones 2011: 850). Thibaud (2001) spricht auch von „parcours commentés“ im Sinne von Rundgängen, in denen die Teilnehmenden das im Gehen Vorgefun- 
Tabelle 1: Die drei Dimensionen der Raumproduktion nach Lefèbvre

\begin{tabular}{llll}
\hline Produktionsprozess & $\begin{array}{l}\text { Sprachtheoretische Spezi- } \\
\text { fikation }\end{array}$ & $\begin{array}{l}\text { Phänomenologische Spezi- } \\
\text { fikation }\end{array}$ & Produkt \\
\hline Materielle Produktion & Räumliche Praxis & Wahrgenommener Raum & Materialität \\
Wissensproduktion & Repräsentation des Raumes & Konzipierter Raum & Diskursive Konzepte \\
Bedeutungsproduktion & Räume der Repräsentation & Erlebter Raum & Symboliken \\
\hline
\end{tabular}

Quelle: Eigene Darstellung aufbauend auf Lefèbvre (1991)

dene kommentieren. Für Fragen der Stadtentwicklung etwa begleiten Forschende Bewohner des Stadtraums auf ihren alltäglichen Wegen, während die Teilnehmenden ihre Perspektive auf den Stadtraum darlegen. Nach ähnlichen Vorstellungen eines Dialogs mit der Bürgerschaft begründete Burckhardt (2006) seinen Ansatz der Promenadologie. Dabei ähneln Walking Interviews Partizipationsmethoden der räumlichen Planung, wie geführte Rundgänge oder „Transect Walks“ (Chambers 1997). Diese Prozesse erfolgen in der Regel in Gruppen, meist bestehend aus Privatpersonen und Experten, und dienen der Herstellung eines Dialogs über den Planungsraum. In gemeinsamen Rundgängen werden Meinungen, Anregungen und Kritik ausgetauscht und diskutiert. Damit ließe sich die Methode auch als mobile Fokusgruppe charakterisieren. Im Gegensatz hierzu bilden Walking Interviews eine empirische Methode, die eine Erkenntnisbasis für partizipative Planungsprozesse liefern kann. Walking Interviews werden eingesetzt, um individuelle Aneignungen des Stadtraums methodisch kontrolliert herauszuarbeiten. Dabei strebt die Erhebung nicht explizit einen Dialog als Teil der Partizipation an, sondern blickt auf Aneignungsprozesse, wie sie sich im gelebten Alltag individuell vollziehen.

Walking Interviews dienen originär der Erhebung von Wahrnehmungsweisen im phänomenologischen Sinne. In einer praxistheoretischen Anwendung ermöglichen Walking Interviews das Erfassen von Praktiken im Kontext ihres Vollzugs. So werden planungsrelevante Belange unmittelbar entlang der praktischen Alltagsgestaltung im Planungsraum aufgearbeitet. Anhand der beobachteten Praktiken im Rahmen der Walking Interviews werden alltägliche Raumproduktionen in situ empirisch zugänglich. Dadurch wird ersichtlich, wie sich Menschen im Raum bewegen, diesen wahrnehmen, sich mit Räumen auseinandersetzen und durch inr Tun (mit)gestalten (Herbert 2000: 551). Zugleich ist auch der Vollzug des Interviews selbst als Praktik zu begreifen. Ist es den Interviewten überlassen, welche Aspekte sie im Walking Interview aufgreifen, zeigen sich entlang der Auswahl dieser Aspekte und in der Art ihrer Darlegung charakteristische Relevanzsetzungen der Interviewten in der Auseinandersetzung mit ihrer Umwelt (Kusenbach 2003: 466). Dabei wird anhand von Sprech- und Argumentationsweisen deutlich, wie sozial geteilte Wahrheiten als Teil des konzipierten Raums die Erfahrenswirklichkeit mit ausgestalten. Ebenso zeigt sich in Gesprächen über Dinge und Geschehnisse vor Ort sowie über Erinnerungen, die an Räume geknüpft sind, wie sich Menschen von ihrer Umwelt angesprochen fühlen und welche Konsequenzen sich hieraus für die Aneignung des Stadtraums im Sinne eines gelebten Raumes ergeben. In dieser Aufarbeitung von Praktiken der Raumproduktion wird schließlich das Spektrum sichtbar, in dem dieselben Räume nach verschiedenen Relevanzsetzungen praktisch angeeignet werden. Unter innen bilden sich überindividuelle Gemeinsamkeiten ab, die sich als typische Ausprägungen bündeln lassen.

\subsection{Erkenntnisse für die Planung}

Mit der Identifikation typischer Relevanzsetzungen erschließen sich Anhaltspunkte, wie Städte nach den Vorstellungen der Bürgerschaft alltagsgerecht zu gestalten sind. Praxistheoretisch gewandt lässt sich schlussfolgern, wie eine Planung für Praktiken gelingt, als eine Planung, die den praktischen Vollzug des Alltagslebens gemäß der identifizierten Relevanzsetzungen ermöglicht. So können sich planerische Abwägungen an den verschiedenen Erfahrungswirklichkeiten und den hiermit einhergehenden differenzierten Relevanzsetzungen orientieren. Dabei eignet sich das Verfahren für die Konzeption neuer Stadtquartiere ebenso wie für Stadtentwicklungsvorhaben in bestehenden Siedlungsstrukturen, in denen eine bauliche Erweiterung, Umgestaltung oder Aufwertung vorgenommen werden soll. Hier lässt sich erkennen, wie Stadträume und Infrastrukturen zu entwickeln sind, damit sich Menschen hiervon angesprochen fühlen. In Anwendung entspre- 
chender Erhebungen werden dabei gerade nicht finale Planungen mit wenigen aktiven Bürgern diskutiert, wie etwa in informellen Beteiligungsverfahren üblich. Stattdessen können die Belange bereits zuvor als Orientierung und Richtmaß des Planentwurfs dienen. So bietet ein differenziertes Wissen über die vielfältigen Vorstellungen und Bedarfe eine Hilfestellung auf der Suche nach geeigneten planerischen Lösungen (vgl. auch Huning 2004). Konfliktpotenziale divergierender Relevanzsetzungen werden sichtbar und können offensiv angegangen werden. Ebenso erleichtern die genauen Kenntnisse der unterschiedlichen Belange die Suche nach Lösungen, die den Vorstellungen und Bedarfen möglichst vieler gerecht werden. Dabei werden die identifizierten Bedarfe nicht an soziodemographischen Merkmalen festgemacht, sondern induktiv abgeleitet. Mittels geschichteter Stichprobenziehung innerhalb empirischer Erhebungen können verschiedene soziale Gruppen in methodisch kontrollierter Form zu Wort kommen, hierunter insbesondere auch jene, die sich aus verschiedenen Gründen nicht an Partizipationsverfahren beteiligen (vgl. Nolte 2011). Damit lässt sich die empirische Ermittlung von Belangen als eine Form der Partizipation verstehen, in der Bürger als Expertengruppe des lokalen Alltagslebens konsultiert werden. Die Forschenden tragen hier die Verantwortung, die Erhebung so zu gestalten, dass vernachlässigte Interessengruppen in die Erhebung nach Möglichkeit eingeschlossen werden. Dabei ist nach Burckhardt (2004: 85) „die Fähigkeit zur Artikulation der eigenen Bedingungen überall vorhanden. Wo Schwierigkeiten auftreten, handelt es sich um Übersetzungsfragen: Die Beplanten leben in ihrer Realität, ebenso auch die Planungsberechtigten in der ihren". Für diesen Übersetzungsprozess wiederum sind niederschwellige Verfahren zur Erhebung verschiedenster Sichtweisen und Belange dem Vorbild der Gemeinwesenarbeit anschlussfähig (vgl. Gerlich/Stoik 2008). Um die Barriere zwischen Forschenden bzw. Planenden und der Bürgerschaft gering zu halten und Anliegen, Problemlagen und Vorzüge des Lebensraumes alltagsnah zu erschließen, werden ebenso alltagsnahe Kommunikationssituationen gesucht (Gerlich/Stoik 2008: 257). Hier greift der methodische Zugang des gemeinsamen Gehens durch den Stadtraum auf eine vertraute Praktik zurück, in der verschiedene Aneignungsformen der alltäglichen Umwelt in Augenschein genommen werden können, um so individuellen Sichtweisen auf Planungsräumen näherzukommen.

\section{Das Beispiel „Grün im städti- schen Alltag“"}

Im Folgenden soll beispielhaft aufgezeigt werden, wie differenzierte empirische Erkenntnisse über alltagsweltliche Sichtweisen auf Stadträume eine Orientierung für planerische Abwägungen bieten können, in denen Belange der Bürgerschaft in den Fokus rücken. Hierzu wird auf eine Studie von Kühl (2016b) zurückgegriffen, die sich speziell der Relevanz städtischen Grüns (im Sinne von Begrünung, Grünflächen, Anpflanzungen) widmet. Mit diesem inhaltlichen Fokus sind die Erkenntnisse für Fragen der Grünplanung anschlussfähig. Ebenso lassen sie Schlüsse über Potenziale zur Aufwertung von Stadträumen sowie über die Bewertung von Vorhaben aus der Sicht unterschiedlicher Bürger zu.

\subsection{Kontext und Methodik}

Die Studie arbeitet die Bedeutung von Grün für ein Wohnen und Leben in Stadträumen praxistheoretisch auf. Dabei ist „Grün“ als ein Signifikant für all jenes zu verstehen, was Menschen individuell als Grün deuten und verstehen. Dieses möglichst offene Begriffsverständnis soll helfen, eine explizit alltagsweltliche Bedachung von Phänomenen städtischen Grüns zu verfolgen, anstatt das Phänomen selbst durch die Verwendung von Kategorien wie grüne Infrastrukturen oder städtische Grünflächen zu überdecken bzw. in planerischer Logik ,vorzukonstruieren'. So soll unter dem Signifikant gerade herausgestellt werden, was Begrünung und Grünformen jenseits planerischer Kategorien ausmachen und in welchen Ausprägungen sie Relevanz erlangen.

Im Rahmen der Studie wurden 29 Walking Interviews mit Bewohnern zweier zentrumsnaher, urban geprägter Quartiere in Kiel (Blücherplatz) und Dortmund (Kreuzviertel) durchgeführt. Die Stadtquartiere wurden als Kontext alltäglicher Raumproduktionen gewählt, während sich die alltäglichen Praktiken selbstverständlich hierüber hinaus erstrecken (können). Die bauliche Gestalt beider Quartiere ist von einem dichten Geschosswohnungsbau in Blockrandbebauung mit einem hohen Anteil an Gründerzeitbeständen geprägt. Daneben verfügen beide Quartiere über ein ähnliches Repertoire sowie über einen ähnlichen Umfang an Grünangeboten. Die Interviewteilnehmenden wurden mittels öffentlicher Aushänge zur Teilnahme eingeladen. Um einen möglichst breiten Kreis an Interviewpartnern zu generieren, erfolgte zusätzlich eine gezielte Akquise über soziale 
Kontakte, die Teilnehmende vermittelten, sowie durch die Ansprache von Bewohnern des Quartiers.

Aufbauend auf der Betrachtung von Quartieren als Kontext und Gegenstand alltäglicher Raumproduktionen nach Lefèbvre wurde ein Forschungsdesign verwendet, das alle drei Dimensionen der Raumproduktion einschließt (vgl. Kühl 2016a; Tabelle 1). Die Erhebung begann für jeden Teilnehmenden mit einem Leitfadeninterview, an das sich unmittelbar ein Walking Interview anschloss. Während der Walking Interviews erstellten die Teilnehmenden zudem Fotodokumentationen ihrer Umwelt. Mithilfe der Leitfadeninterviews wurde ermittelt, welchen objektiven Konzepten das Tun folgt. Anhand der Walking Interviews wurden Ausprägungen einer räumlichen Praxis erkennbar, in denen der Stadtraum physisch wahrgenommen und reproduziert wird. Ebenso wurden mit dem gemeinsamen Gehen bedeutungsgeladene Räume der Repräsentation identifiziert, die bestimmte Erlebensweisen symbolisieren. Gleichzeitig halfen Fotografien dabei, individuelle Erlebensweisen von Stadträumen zu dokumentieren. Sind die Teilnehmenden angehalten, während der Walking Interviews Orte und Entitäten zu fotografieren, die für sie persönlich bedeutsam sind, lassen sich auf diese Weise spezifische Bedeutsamkeiten im Stadtraum aufdecken.

Zur Identifikation von Relevanzsetzungen eignen sich Analysemethoden der rekonstruktiven Sozialforschung, wie insbesondere die dokumentarische Methode (vgl. Bohnsack 2010; Nohl 2012). Sie bieten eine strukturierte Vorgehensweise zur sequenziellen Analyse der Art und Weise, wie im Wahrnehmen, Erleben und Konzipieren Erfahrungswirklichkeiten von Räumen geformt werden. Ebenso zeigen sich Regelmäßigkeiten als sozial geteilte Relevanzsetzungen, nach denen Menschen den Stadtraum erschließen. Hierauf aufbauend wurde das Interviewmaterial transkribiert und mithilfe der dokumentarischen Foto- und Textinterpretation analysiert. Dabei wurde herausgearbeitet, in welcher Weise Menschen ihren Stadtraum über die Sinne wahrnehmen und physisch mit inm umgehen (Räumliche Praxis I Wahrgenommener Raum). Gleichermaßen wurde nachvollzogen, welchen sozial geteilten Konzepten die Menschen dabei folgen und welche Wertvorstellungen sie formulieren (Repräsentationen des Raumes / Konzipierter Raum). Hierzu zählen beispielsweise objektivierende Konzepte und Faktenwissen als geltende Wahrheiten sowie normative Vorstellungen von Natur und Gesellschaft. Zudem wurde betrachtet, wie Menschen inre Umwelt individuell erleben und aufgrund dieses Erlebens Bedeutungen zuschreiben (Räume der Repräsentation / Gelebter Raum). Als Ergebnis der Analyse wurden mittels komparativer Verfahren der dokumentarischen Typenbildung überindividuelle Ausprägungen von Relevanzsetzungen zu typischen Modi der Raumproduktion abstrahiert. Sie beschreiben, in welcher sinnhaften Weise sich Menschen Stadträume praktisch aneignen und inwiefern sie dabei Entitäten und Situationen Relevanz beimessen.

\subsection{Empirische Ergebnisse}

Für die betrachtete Fallstudie lassen sich acht typische Modi identifizieren. ${ }^{1}$ In Abstraktion idealtypischer Ausprägungen alltäglicher Praktiken der Raumproduktion können die Modi wie folgt zusammenfassend charakterisiert werden (vgl. Kühl 2016b: 170 f.):

- M1: Grün ist erfüllend, wenn es einen Nutzen erfüllt: Im Modus Zweckausrichtung ist der praktische Zweck der Grünnutzung zentral, während ein gegebener Nutzen als elementar für die Lebensqualität im Alltag erlebt wird.

- M2: Grün unterstreicht eine schöne heile Welt: Im Modus der Behaglichkeit ist Grün ein Ausstattungsmerkmal, das zu einer heimeligen Umwelt beiträgt.

- M3: Besonders Grün und besonderes Grün für besondere Ansprüche: Im Modus Privilegiert Leben unterstreicht die Qualität und Quantität an verfügbarem Grün den sozialen Status, während diese Verfügbarkeit von Grün zugleich besondere Vorzüge bietet.

- M4: Ist das Grün in bester Ordnung, so ist alles in Ordnung: Im Modus Kontrollieren und Evaluieren ist Grün ein Spiegel des sozialen Umfeldes, wobei sich über ein ,kontrolliertes', gepflegtes Grün eine redliche Nachbarschaft ausdrückt.

- M5: Freude am Angenehmen und an einem angenehmen Miteinander: Im Modus Ergötzen eröffnen schöne Eindrücke ein Wohlbefinden und drücken eine angenehme Gemeinschaft aus.

- M6: Verteidigen von Grün für das persönliche Seelenheil: Im Modus Patronisieren steht Grün in Abgrenzung zum Selbstverständnis der Gesellschaft für die Herstellung eines inneren Einklangs mithilfe der Natur.

- M7: In der Natur und mit der Natur leben: Im Modus Naturbesinnen dient Grün als Zugang zur Natur, mit dem sich neue Perspektiven erschließen und der ein Wohlbefinden auch in der Stadt ermöglicht.

1 Eine ausführliche Darstellung der Modi findet sich bei Kühl (2016b). 
- M8: Grün hält Entdeckungen abseits des städtischen Pflasters bereit: Im Modus Entdecken dient Grün als eine Nische im Stadtraum, in der Natur erlebt werden kann.

Diese Abstraktion der Modi zeigt die Varianz der Erfahrungswirklichkeiten von Grün. Zur Konkretisierung der Modi werden im Folgenden die Modi M4 und M6 exemplarisch zusammenfassend dargestellt. ${ }^{2}$

\subsubsection{Beispiel M4: Kontrollieren und Evaluieren}

In diesem Modus bestehen genaue Kenntnisse über den Stadtraum und seine Bewohnerschaft. Veränderungen werden rasch wahrgenommen und mit kritischer Haltung bewertet. Immer wieder richtet sich die Wahrnehmung auf Unzulänglichkeiten im Stadtraum, an denen man sich stört. Als maßgebend dient dabei ein Selbstverständnis, nach dem eine Nachbarschaft schön und gepflegt zu sein hat. Ebendies wird vor allem auch über Grün inszeniert. Dabei wird Grün als Anzeiger sozialer Verhältnisse und zu einem Symbol von Redlichkeit aufgegriffen.

In diesem Modus sind größere Parkanlagen im näheren Umfeld zwar bekannt, jedoch im Alltag kaum relevant. Demgegenüber sind Straßenzüge mit bepflanzten Vorgärten und Straßenbäumen sowie grüne Plätze und Hinterhöfe als schöne Ansichten präsent. Auch werden wohnstandortnahe Grünflächen und Plätze als Orte zum Verweilen geschätzt - jedoch nur, wenn sie sich in einem gepflegten Zustand befinden. Andernfalls führen sie zu Unmut und Empörung. So wird etwa eine Baumscheibe mit Unkraut zum Ärgernis, da sich offenbar niemand in erwarteter Weise kümmert und in der Folge ein unansehnlicher beschämender Anblick haften bleibt (vgl. Abbildung 1). Es wird lieber auf Grün verzichtet, als ungepflegtes Grün hinnehmen zu müssen. In der Unordnung drückt sich die Ignoranz und Untätigkeit der Mitmenschen und auch der Stadtverwaltung aus, während man andere in der Pflicht sieht, diese Ordnung zu wahren. Gepflegtes Grün wiederum schafft Ordnung und lässt die Umgebung als eine redliche Nachbarschaft erscheinen. Dabei ist Grün vor allem in symmetrisch und ästhetisch perfektionierter Form als raumstrukturierendes Element, wie etwa anhand Abbildung 2 dokumentiert, gewünscht.

Beispielreferenz aus einem Walking Interview (Kiel, Blücherplatz):

2 Alle im Folgenden genannten Namen wurden zu Anonymisierungszwecken geändert.

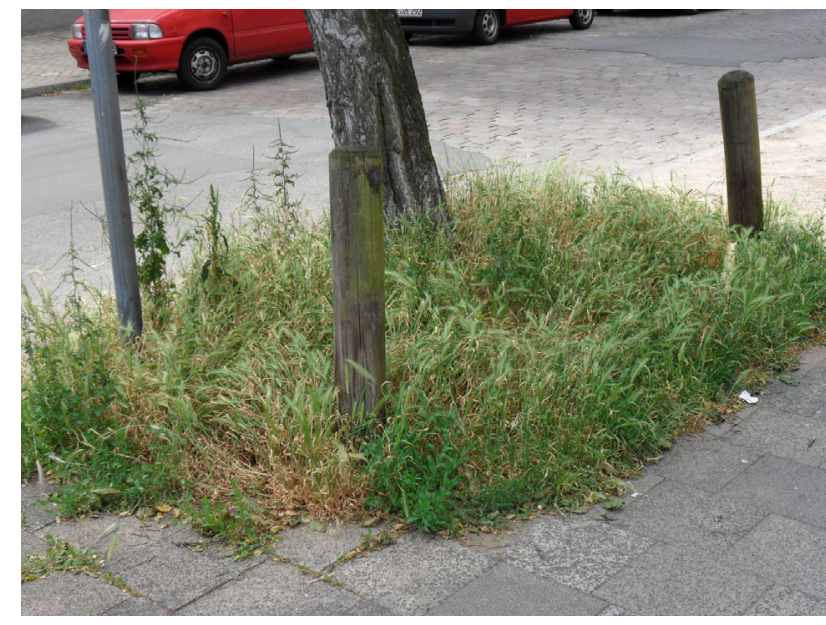

Abbildung 1: Eine Baumscheibe als Ärgernis

Aufnahme der Interviewteilnehmerin Sabine im Rahmen des Walking Interviews

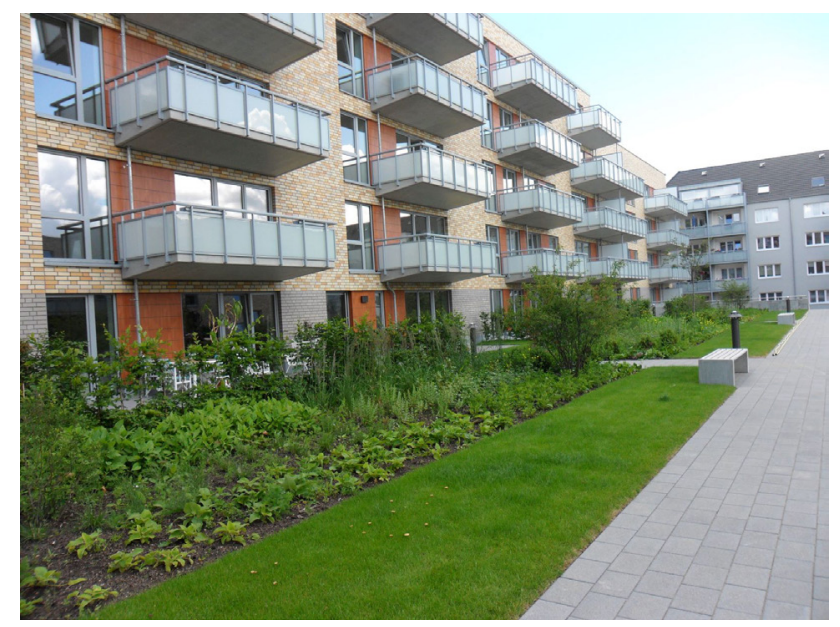

Abbildung 2: Ein Hinterhof: ordentlich, klar gegliedert und ansehnlich

Aufnahme der Interviewteilnehmerin Sabine im Rahmen des Walking Interviews

Sabine betont, dass die richtige Gestaltung von Grün wichtig ist für die Aufenthaltsqualität. Sie möchte Grün ebenso wie Cafés als Setting zum Verweilen nutzen. Grün erscheint als ein Angebot, das bei entsprechender Attraktivität konsumiert wird. Dabei dienen Grünelemente der Herstellung einer klaren Raumgliederung und als Dekoration („Blumiges“ als „Farbklecks“). Grün ist nicht selbstverständlich Teil des Stadtraums, sondern es wird platziert und dekorativ geformt. Damit Grün die dekorativen Zwecke erfüllt, ist die Grünpflege zentral. Es wird selbstverständlich angenommen, dass alle Nachbarn den Wunsch nach gepflegtem Grün teilen. Zugleich nimmt man sie in die Pflicht, durch die Grünpflege aktiv zu einem ordentlichen Stadtbild beizutragen. Die Herstellung eines geordneten Grüns erscheint hier als bedingungslos. 
Autorin: Jetzt muss ich nochmal nachfragen: Wo fängt denn für dich persönlich Grün an? Was kannst du als Grün akzeptieren?

Sabine: Unter Grün versteh ich einen schönen Baum mit einer Sitzgelegenheit darunter, vielleicht noch ein paar äh so eine kleine Hecke, einige eingefasst, und ein bisschen was Blumiges. Wir brauchen einen Farbklecks. Das ist für mich Grün.

Autorin: Okay, also der Baum allein tut's nicht?

Sabine: Nee! Also der ist zwar schon mal nicht schlecht, ist aber, das ist ein guter Anfang, sag'ich mal. Ich mein' man will ja was davon haben, ne?! Ähm, jetzt hier gerade auch an der B.-Straße äh da find ich das total schön, dass wir hier überall in Abständen diese die Bäume haben. Find ich wichtig. Da ist allerdings kein Platz zum Sitzen. Obwohl viele dann ihre Dinger rausstellen, ihre Tische und Stühle, aber ähm nee, also Grün also von der Optik her darf es gerne grün sein aber um richtig Grün erleben zu können, muss man eben etwas mehr davon haben. Und vor allen Dingen es muss gepflegt werden. Das find ich auch ein großes Problem. Ist immer gut gemeint aber dann äh wird man da alleine gelassen und das ist auch eine schöne Idee die ich mir überlegt hab. (lacht) So Patenschaften zu übernehmen, ne? So dass sozusagen immer Hausgemeinschaften oder äh vor allen Dingen auch viele Ältere, die vielleicht früher mal einen Garten hatten oder so, dann in die Stadt ziehen und dann schade finden, dass sie kein Grün haben, dass man sozusagen Patenschaften übernimmt für einzelne Bäume, so ähnlich wie man das mit den Hundeschietbeuteln hat, wo man immer mal ein Auge drauf wirft, und es muss ja nicht bepflanzt werden aber zumindest von Unkraut befreien und so ein bisschen Aufhacken oder irgendwie sowas. Ich glaube, da wäre schon viel getan.

\subsubsection{Beispiel M6: Modus des Patronisierens}

Im Modus des Patronisierens wird die Umgebung mit wachen Sinnen erfasst und die Wirkung der Sinneseindrücke reflektiert. Über Grün in der Stadt lässt sich ein Bezug zur Natur herstellen, der in der Stadt verlorenzugehen droht. Dabei wird Grün als Ausgleich zu dem Umweltstress der Stadt gezielt gesucht. Je größer und höher die Grünformen, desto kleiner und unbedeutender lassen sie die gebaute Stadt erscheinen. Graue Fassaden, laute Straßen wie auch Unästhetisches im Stadtraum werden durch Grün erträglicher. Begrünung und Grünräume werden spirituell anmutend als eine Quelle des eigenen Wohlbefindens und des persönlichen Einklangs erlebt. Doch treten sogleich anhand des Umgangs der Menschen mit Grün gesellschaftliche Widersprüche hervor. Es herrscht ein Unverständnis darüber, dass andere die Bedeutung von Grün offenbar verkennen und es beseitigen. Im Beispiel von Abbildung 3 etwa wurde ein ehemals begrünter Vorgarten zu einem Parkplatz und Müllplatz umgewandelt. Das Foto repräsentiert einen belastenden Anblick, der sich ergibt, wenn Grün fehlt. Zugleich drückt sich hierin das Unverständnis über die Mitbürger aus, die dies verantworten. Durch die Beseitigung von Grün wächst ein Ungleichgewicht von gebauter Stadt und Natur und der Stadtraum wird unwirtlich. Wie auch im Modus des Kontrollierens und Evaluierens werden Veränderungen genau registriert, jedoch ist diese Beobachtung von der Vorstellung geleitet, so viel Natur wie möglich in der Stadt erleben zu können. Auch wird Grün nicht wie im Modus des Kontrollierens und Evaluierens als Dekoration verstanden, sondern als lebende Kreatur, dessen Daseinsberechtigung im Stadtraum es zu verteidigen gilt. Wer Grün beseitigt, wird zum Widersacher und man selbst sorgt sich aktiv darum, Grün in der Stadt auszubreiten. Ein Beispiel hierfür findet sich in Abbildung 4. Das Bild zeigt eine Garagenwand, die einst mit Efeu bewachsen war. Für die Interviewpartnerin, die das Foto machte, ist es unverständlich und ärgerlich, dass der Efeu entfernt wurde, da anstelle von Grün nun ein hässlicher Anblick zum Vorschein tritt, der die Betrachterin in ihrer Befindlichkeit stört. Die Garagenwand wird zum Sinnbild des Ringens um Grün in der Stadt für das eigene Wohlbefinden. Doch wird Grün dabei nur so lange geschätzt, wie es zu dem eigenen Wohlbefinden beiträgt.

Beispielreferenz aus einem Walking Interview (Dortmund, Kreuzviertel):

Frau Winter möchte, anders als ihre Nachbarn, die Versiegelung von Vorgärten rückgängig machen und Grün mehr Raum geben. Eine entsiegelte Garage wird so zum Sinnbild grüner Stadträume. Frau Winter braucht das Grün, damit sie sich wohlfühlen kann. Sie schafft sich im Privaten bewusst ihr eigenes Grün als eine Gegenwelt, die ihrem Bedürfnis nach Grün gerecht wird. Auch verschafft ihr diese Praxis eine Genugtuung, der Dezimierung von Grün in der Stadt entgegenzuwirken.

Autorin: Als allererstes würde mich mal interessieren, was verbinden Sie spontan ähm mit dem Begriff Grün in der Stadt?

Frau Winter: Kastanien, Platanen und eben diese Vorgärten und dass man eben auch ja an den Häusern auch Grün hat. Also wenn's nach mir ginge, dann würde an den Häusern einfach mehr hochwachsen. Ich habe auch hier, das war ja eine Garage, die überhaupt nicht benutzbar war. Und dann habe ich einfach ja also den Vermieter überredet, dass man das Dach abmacht und da habe ich einen Garten reingemacht, weil mir das einfach total wichtig ist, einfach so ein bisschen Grün vor der Tür zu haben. Haben Sie ja bestimmt auch gemerkt, wenn man hier drauf zukommt, das ist mir 


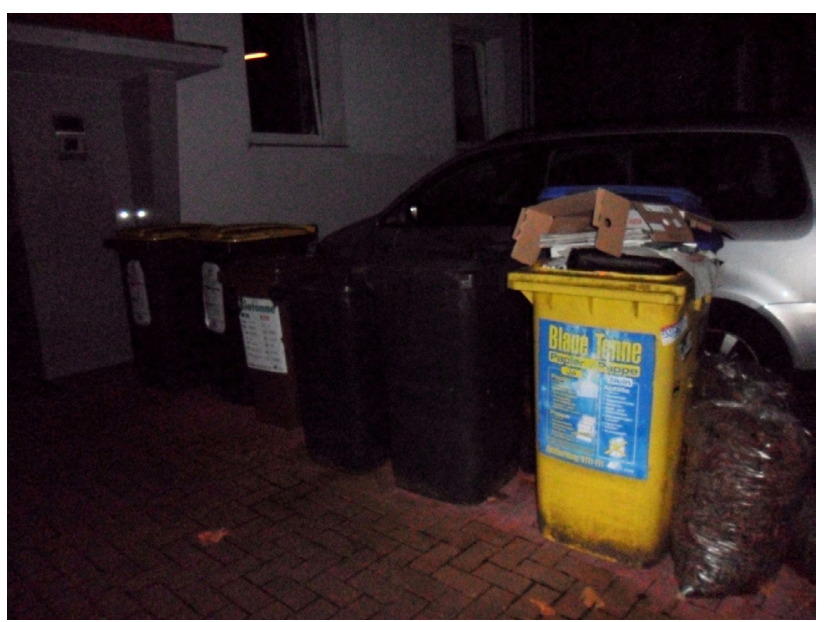

Abbildung 3: Versiegelter Vorgarten anstelle von Grün Aufnahme der Interviewteilnehmerin Frau Winter im Rahmen des Walking Interviews

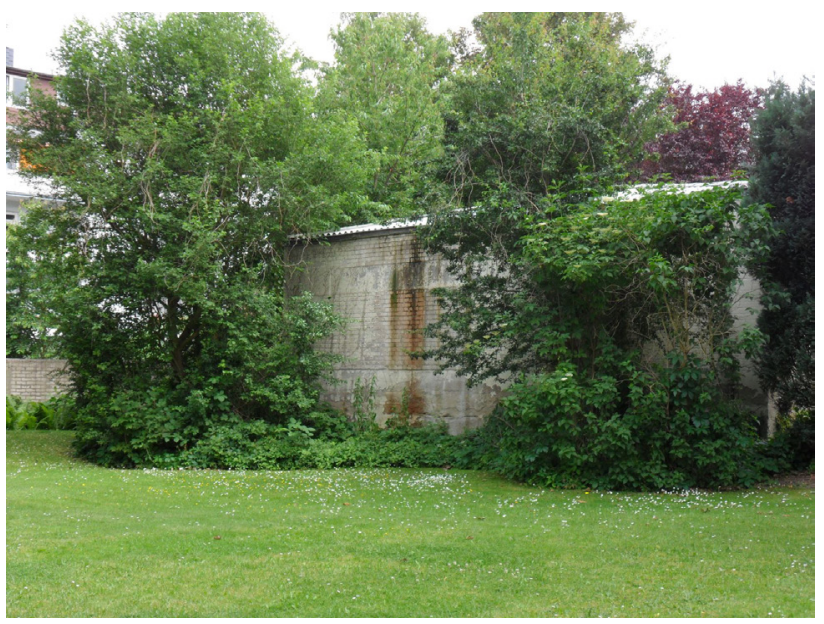

Abbildung 4: Unansehnliche Garagenwand im Innenhof Aufnahme der Interviewteilnehmerin Lena im Rahmen des Walking Interviews

total wichtig. Das ist lebenswert einfach. Wenn mir das Haus gehören würde, dann würde ich auch den Asphalt wegmachen und dann gucken, dass dann auch irgendwie ja das Wasser versickern kann, dass da irgendwie sowas ist, wo was Grünes dazwischen wachsen kann.

Frau Winter verbindet mit Grün große, vertikale Grünformen. Die Bäume relativeren menschliche Raumaneignungen. Ihr Anblick fördert Glück und Wohlbefinden.

Autorin: Was denken Sie, wenn Sie sich damit [gemeint ist: mit Grün] auseinandersetzen?
Frau Winter: Ach das sind dann so Momente, wo man richtig sogar entspannen kann. Also wenn ich irgendwie über den C.-Platz geh' und nach oben gucke und irgendwie guck', wie die Blätter gerade aussehen und da fällt das Licht durch, das ist Glück. Oder wenn man irgendwie da vorne steht an der K.-Straße, ne das ist die W.-Straße und dann irgendwie da hochschaut und diese ganzen Platanen sieht, das ist einfach auch ein schöner Anblick, find ich. Und da kommen einem die Autos dann ganz klein vor und winzig irgendwie.

\subsection{Erkenntnisse für die Planung von Grün in Stadträumen}

Mit der dargelegten Erhebung wird das Spektrum, in dem Grün im Altäglichen Relevanz zukommt, aufgezeigt und anhand typischer Ausprägungen zusammengefasst. Hiervon lässt sich ableiten, wie Grün im Stadtraum gemäß verschiedener Relevanzsetzungen der Bürger planerisch entwickelt werden sollte. Ebenso wird erkennbar, wie mithilfe einer bestimmten Gestaltung von Grün im Stadtraum Bürger gezielt angesprochen werden können. Dabei lassen sich die identifizierten Belange, wie etwa Erwartungen, wie Grün im Stadtraum auszusehen hat, in ihren Sinnzusammenhängen verstehen. So kann etwa ein Park zum Joggen aufgesucht werden. Jedoch verbindet der oder die Einzelne mit diesem Besuch Unterschiedliches und stellt auch unterschiedliche Anforderungen an einen Park. Jemand mag den Park als nächstgelegene Trainingsgelegenheit aufsuchen, während ebenso ein Sportplatz geeignet ist (Modus der Zweckausrichtung). Oder aber der Park erscheint aufgrund der grünen Umgebung als Kontrastort zum bebauten Stadtraum, an dem das Joggen erst die gewünschte Entspannung eröffnet (Modus des Patronisierens). Ebenso sind je nachdem, ob Grün für ein Naturerleben, als Nutzungsraum oder als Anzeiger sozialer Ordnung aufgegriffen wird, andere Formen von Grün relevant.

Wird das Wissen über die unterschiedlichen Erfahrungswirklichkeiten planerisch aufgegriffen, werden im Ergebnis nicht allein Funktionalitäten bereitgestellt, wie etwa ein Park als Erholungsraum. Vielmehr erlangt die Planung Anhaltspunkte, um Relevanzsetzungen gezielt anzusprechen. So können etwa Relevanzsetzungen im Modus des Kontrollierens und Evaluierens wie auch im Modus der Behaglichkeit über ein gepflegtes und geordnetes Grün angesprochen werden. Im Modus Privilegiert Leben bilden sichtbare Qualitäten des vorhandenen Grüns, die auf eine privilegierte Wohnsituation verweisen, ein wichtiges Merkmal. In den Modi Naturbesin- 
nen, Entdecken und Patronisieren werden Stadträume geschätzt, in denen Grün den Anschein einer Natürlichkeit im Sinne einer Unberührtheit weckt. Hier machen größere Grünformen und ausreichend Grünwachstum Städte lebenswert. Im Modus Zweckausrichtung sind es die Möglichkeiten, die sich durch Grünangebote bieten, die zu einer Zufriedenheit im Alltag beitragen. Ebenso können die Relevanzsetzungen Anhaltspunkte liefern, um Praktiken in einer planerisch angestrebten Weise zu ermöglichen. Ist etwa mehr Radverkehr gewünscht, kann das Säumen von Radwegen mit Grün dazu beitragen, dass Radwege vermehrt genutzt werden, da das Grün eine sichere Distanz zum Autoverkehr herstellt (insbesondere Modus der Behaglichkeit) und die grünen Eindrücke das Radfahren angenehmer machen (insbesondere Modus Patronisieren). Gleichermaßen lassen sich mithilfe der Erkenntnisse der Modi sozialen Problemlagen der Stadtentwicklung strategisch begegnen. Zunächst wertet bereits das Vorhandensein von Grün den Stadtraum als Kontrast zur gebauten Stadt auf. Gleichzeitig dient Grün als Repräsentation des Sozialen. Ist das Grün gepflegt und geordnet, erscheinen auch die sozialen Verhältnisse in der Nachbarschaft geordnet (Modus Kontrollieren und Evaluieren). Zudem können gärtnerische Bemühungen von Bürgern als Symbolisierungen eines wertschätzenden Miteinanders von Nachbarschaften dienen (Modus Ergötzen). Ebenso lässt sich mithilfe von Grün eine gute Wohnlage inszenieren, indem ein hoher Grünanteil sowie gepflegte Grünformen als Symbole einer guten Situiertheit instrumentalisiert werden (Modus Privilegiert Leben).

\section{Fazit}

Eine praxistheoretische Aufarbeitung von Belangen der Bürgerschaft, wie sie aus dem Alltagsleben hervorgehen, liefert Orientierung für eine Planung für Praktiken, mit der sich Stadträume nach alltagspraktischen Kriterien gestalten lassen. Die Abstraktion typischer Modi der Raumproduktion verdeutlicht, was es aus der Sicht der Bevölkerung braucht, um den Alltag in gewünschter Weise praktisch zu gestalten und sich in einer ansprechenden Umwelt zu bewegen. Ziel ist es hier, Belange in ihrem Sinnzusammenhang zu erkennen und zusammenzufassen. Die vorgestellte empirische Betrachtung erschließt ein breites Spektrum unterschiedlichster Belange und arbeitet sie systematisierend auf, um so planerischen Abwägungen eine Orientierung zu bieten. Mit diesen Erkenntnissen werden die Grundvoraussetzungen für eine sozial integrative Planung geschaffen, die einen möglichst weitreichenden Konsens mit den Belangen der Bürgerschaft anstrebt. Hierzu gehört auch, Ängste, Befürchtungen und Vorwürfe aufzunehmen und diesen nachzugehen. Nur wenn die konkreten Belange wie auch hiermit verbundene Missgünste bekannt sind, lassen sich Proteste entkräften und Lösungswege nach einem demokratischen Grundverständnis beschreiten. Hier ist beiderseits eine engere Verzahnung zwischen sozialwissenschaftlicher Forschung und Planungspraxis gefragt, um ein genaueres Wissen über soziale Zusammenhänge zur Bewältigung planerischer Herausforderungen zu nutzen. Empirische Erhebungen, wie die in diesem Beitrag dargestellte, schaffen eine Basis für planerische Abwägungen. Auch lassen sich die für einen soziokulturellen und stadtstrukturellen Kontext ermittelten Vorstellungen und Bedarfe auf andere vergleichbare Ausschnitte von Stadträumen und Stadtgesellschaften übertragen. Während nicht jedes Planungsvorhaben von einer empirischen Studie begleitet werden kann, ist hier die Entwicklung von Erhebungs- und Analysesets sinnvoll, mit denen typische Relevanzsetzungen als Basis planerischer Abwägungen ermittelt werden können. Gleichzeitig liegt es jedoch in der Hand der Planenden, derartige Grundlagen zu suchen und zu nutzen. Ebenso bleibt offen, wie unterschiedliche Belange in die Planung einfließen und inwiefern Belange sozial gerecht zum Ausgleich gebracht werden. Hier eröffnet sich ein komplexes Forschungsfeld: „The project for many progressive planners has been to improve the liveability and sustainability of the conditions of life of the diverse many and not just the few, in a world increasingly recognized in its complex multiplicities, with competing and conflicting structuring dynamics and power relations" (Healey 2012: 61). Die Abwägung der unterschiedlichen, oft widersprüchlichen Belange ist ein schwieriger Prozess, der einer dezidierten Betrachtung bedarf. Hierunter drohen immer auch Machtstrukturen und Dogmen der Planungspraxis, die Dominanz ausgewählter Interessen fortbestehen zu lassen. Unter anderem braucht es Verfahren, die Abwägungen transparent machen und eine partizipative und integrative Planungspraxis zur Pflicht werden lassen. Entscheidend ist etwa, inwiefern Vertreter (stakeholder) der räumlichen Planung Belange der Bürgerschaft als legitime Gestaltungsmaßstäbe wahrnehmen und Vetostimmen Entscheidungsmacht einräumen. 


\section{Literatur}

Bergold, J. B.; Thomas, S. (2010): Partizipative Forschung. In: Mey, G.; Mruck, K. (Hrsg.): Handbuch Qualitative Forschung in der Psychologie. Wiesbaden, 333-344.

Bock, S.; Reimann, B. (2012): Neue Qualitäten der Bürgerbeteiligung - oder: alter Wein in neuen Schläuchen? In: Beckmann, K. J. (Hrsg.): Bürgerbeteiligung in Kommunen. Anmerkungen aus der Stadtforschung zu einer aktuellen Herausforderung. Berlin, 13-18. = Difu-Impulse 3/2012.

Bohnsack, R. (2010): Rekonstruktive Sozialforschung. Einführung in qualitative Methoden. Opladen.

Bourdieu, P. (1976): Entwurf einer Theorie der Praxis auf der ethnologischen Grundlage der kabylischen Gesellschaft. Frankfurt am Main.

Brenner, N.; Marcuse, P.; Mayer, M. (Hrsg.) (2012): Cities for people, not for profit. Critical urban theory and the right to the city. London/New York.

Burckhardt, L. (2004): Wer plant die Planung? Architektur, Politik und Mensch. Berlin.

Burckhardt, L. (2006): Warum ist Landschaft schön? Die Spaziergangswissenschaft. Berlin.

Chambers, R. (1997): Whose reality counts? Putting the first last. London.

Deutscher Städtetag (2013): Beteiligungskultur in der integrierten Stadtentwicklung. Arbeitspapier der Arbeitsgruppe Bürgerbeteiligung des Deutschen Städtetages. Berlin/Köln.

Diebäcker, M. (2008): Governance und Demokratie. In: Hamedinger, A.; Frey, O.; Dangschat, J. S.; Breitfuss, A. (Hrsg.): Strategieorientierte Planung im kooperativen Staat. Wiesbaden, 266-281. doi: 10.1007/978-3-531-90804-5

Diller, C.; Thaler, T. (2017): Zum Gap zwischen theoriebasierter Planungsforschung und Planungspraxis. Eine Betrachtung weiter Teile des deutschsprachigen planungswissenschaftichen Outputs seit 2003. In: Raumforschung und Raumordnung | Spatial Research and Planning 75, 1, 57-69. doi: 10.1007 | s13147-016-0431-6

Elden, S. (2002): „Es gibt eine Politik des Raumes, weil Raum politisch ist." Henri Lefèbvre und die Produktion des Raumes. In: An Architektur 1, 27-35.

Evans, J.; Jones, P. (2011): The walking interview: Methodology, mobility and place. In: Applied Geography 31, 2, 849-858. doi: 10.1016/j.apgeog.2010.09.005

Fainstein, S. S. (2010): The just city. Ithaca.

Fischer, F.; Gottweis, H. (Hrsg.) (2012): The argumentative turn revisited. Public policy as communicative practice. Durham.

Florida, R. L. (2004): The rise of the creative class. And how it's transforming work, leisure, community and everyday life. New York.

Friedmann, J. (2010): Place and Place-Making in Cities: A Global Perspective. In: Planning Theory and Practice 11, 2, 149-165. doi: 10.1080/14649351003759573

Gehl, J. (2010): Cities for people. Washington DC.

Gerlich, W.; Stoik, C. (2008): Lokale lebensweltnahe Interessen u. gesamtstädtische Entwicklungsprozesse. In: Hamedinger, A.; Frey, O.; Dangschat, J. S.; Breitfuss, A. (Hrsg.): Strategieorientierte Planung im kooperativen Staat. Wiesbaden, 250-265. doi: 10.1007/978-3-531-90804-5
Gottdiener, M. (2002): Ein Marx für unsere Zeit: Henri Lefèbvre und Die Produktion des Raumes. In: An Architektur 1, 22-25.

Gribat, N.; Kadi, J.; Lange, J.; Meubrink, Y.; Müller, J. (2017): Planung als politische Praxis. Zur Einleitung in den Themenschwerpunkt. In: s u b \ u r b a n. zeitschrift für kritische stadtforschung 5, 1/2, 7-20.

Harvey, D. (2008): The Right to the City. In: New Left Review 53, 23-40.

Harvey, D. (2009): Social justice and the city. Athen. = Geographies of Justice and Social Transformation 1.

Healey, P. (1992): Planning through Debate. The Communicative Turn in Planning Theory. In: Town Planning Review 63, 2, 143162.

Healey, P. (1997): Collaborative planning. Shaping places in fragmented societies. Vancouver.

Healey, P. (2010): Making better places. The planning project in the twenty-first century. Basingstoke.

Healey, P. (2012): Performing Place Governance Collaboratively: Planning as a Communicative Process. In: Fischer, F.; Gottweis, H. (Hrsg.): The argumentative turn revisited. Public policy as communicative practice. Durham, 58-82.

Heinemann, A.; Selle, K.; Sutter-Schurr, H. (2008): Anforderungen an Wohnfreiräume. Nachfragepräferenzen und Nutzerinteressen: Was wissen die Fachleute? In: Schmitt, G.; Selle, K. (Hrsg.): Bestand? Perspektiven für das Wohnen in der Stadt. Dortmund, 202-216.

Herbert, S. (2000): For ethnography. In: Progress in Human Geography 24, 4, 550-568. doi: 10.1191/030913200100189102.

Huning, S. (2004): Der Weg ist das Ziel: Planung als gemeinsames Handeln. In: Altrock, U.; Güntner, S.; Huning, S.; Peters, D. (Hrsg.): Perspektiven der Planungstheorie. Berlin, 45-56. = Reihe Planungsrundschau 10.

Huning, S. (2014): Wer plant für wen? Partizipation im Kontext gesellschaftlicher Differenzierung. In: Küpper, P.; Levin-Keitel, M.; Maus, F.; Müller, P.; Reimann, S.; Sondermann, M.; Stock, K.; Wiegand, T. (Hrsg.): Raumentwicklung 3.0 - Gemeinsam die Zukunft der räumlichen Planung gestalten. Hannover, 33-43. = Arbeitsberichte der ARL 8.

Kovács, Z.; Musterd, S. (2013): The Importance of Places and Place Branding. In: Musterd, S.; Kovács, Z. (Hrsg.): Place-making and Policies for Competitive Cities. Chichester, 97-104.

Kühl, J. (2016a): Erschließung alltäglicher Raumproduktionen am Beispiel von „urbanem Grün“. Konzeptionelle Grundlagen und empirische Zugänge. In: Ludwig, J.; Ebner von Eschenbach, M.; Kondratjuk, M. (Hrsg.): Sozialräumliche Forschungsperspektiven. Disziplinäre Ansätze, Zugänge und Handlungsfelder. Opladen, 227-245.

Kühl, J. (2016b): Grün im städtischen Alltag. Rekonstruktion alltäglicher Raumproduktionen und Anknüpfungen für eine partizipative Stadtentwicklung. Dissertation, Technische Universität Dortmund.

Kühl, J. (2016c): Walking Interviews als Methode zur Erhebung alltäglicher Raumproduktionen. In: Europa Regional 23, 2, 35-48.

Kusenbach, M. (2003): Street Phenomenology: The Go-Along as Ethnographic Research Tool. In: Ethnography 4, 3, 455-485. doi: $10.1177 / 146613810343007$

Lange, J.; Müller, J. (Hrsg.) (2016): Wie plant die Planung? Kulturund planungswissenschaftliche Perspektiven auf die Praxis 
räumlicher Planungen. Berlin. = Berliner Blätter, Ethnographische und Ethnologische Beiträge 72.

Lefèbvre, H. (1991): The production of space. Oxford.

Lefèbvre, H. (1996): The Right to the City. In: Lefèbvre, H.; Kofman, E.; Lebas, E. (Hrsg.): Writings on cities. Cambridge, MA, 147159.

Lepofsky, J.; Fraser, J. C. (2003): Building Community Citizens: Claiming the Right to Place-making in the City. In: Urban Studies 40, 1, 127-142. doi: 10.1080/00420980220080201

Levin-Keitel, M.; Othengrafen, F. (2016): Planungskultur - auf der Suche nach einem kontemporären Verständnis räumlicher Planung? In: disP - The Planning Review 52, 4, 76-89. doi: 10.1080/02513625.2016.1273672

Menzl, M. (2014): Nimby-Proteste - Ausdruck neu erwachten Partizipationsinteresses oder eines zerfallenden Gemeinwesens? In: Gestring, N.; Ruhne, R.; Wehrheim, J. (Hrsg.): Stadt und soziale Bewegungen. Wiesbaden, 65-81. doi: 10.1007/978-3658-01398-1

Nohl, A.-M. (2012): Interview und dokumentarische Methode. Anleitungen für die Forschungspraxis. Wiesbaden.

Nolte, P. (2011): Von der repräsentativen zur multiplen Demokratie. In: Aus Politik und Zeitgeschichte 1-2, 5-12.

Pink, S. (2008): Mobilising Visual Ethnography: Making Routes, Making Place and Making Images. In: Forum Qualitative Sozialforschung 9, 3, 36. doi: 10.17169/fqs-9.3.1166

Reckwitz, A. (2003): Grundelemente einer Theorie sozialer Praktiken. Eine sozialtheoretische Perspektive. In: Zeitschrift für Soziologie 32, 4, 282-301.

Schatzki, T. R. (1996): Social practices. A Wittgensteinian approach to human activity and the social. Cambridge.

Schatzki, T. R. (2001): Introduction: Practice Theory. In: Schatzki, T. R.; Knorr-Cetina, K.; von Savigny, E. (Hrsg.): The practice turn in contemporary theory. New York, 1-14.

Schmidt, R. (2012): Soziologie der Praktiken. Konzeptionelle Studien und empirische Analysen. Berlin.

Selle, K. (2007): Bürgerengagement und Bürgerorientierung in Quartier und Stadt. Von der Ausnahme zur Regel? In: pnd online 2, 1-16.

Simonsen, K. (2007): Practice, Spatiality and Embodied Emotions: An Outline of a Geography of Practice. In: Human Affairs 17, 2, 168-181. doi: 10.2478/v10023-007-0015-8

Tessin, W. (2008): Ästhetik des Angenehmen. Städtische Freiräume zwischen professioneller Ästhetik und Laiengeschmack. Wiesbaden.

Thibaud, J.-P. (2001): La méthode des parcours commentés. In: Grosjean, M.; Thibaud, J.-P. (Hrsg.): L'espace urbain en méthodes. Marseille, 79-99.

Wiechmann, T.; Terfrüchte, T. (2013): Akzeptanz regionaler Planungsprozesse und -ergebnisse. In: Baumgart, S.; Terfrüchte, T. (Hrsg.): Zukunft der Regionalplanung in Nordrhein-Westfalen. Hannover, 18-39. = Arbeitsberichte der ARL 6.

Zibell, B. (2017): Planung(skultur)en zwischen Wissenschaft und Praxis. In: disP - The Planning Review 52, 4, 90-97. doi: 10.1080/02513625.2016.1273674 